\title{
GAIA Spontaneous Abortion and Ectopic Pregnancy Level of Diagnostic Certainty Terminology
}

National Cancer Institute

\section{Source}

National Cancer Institute. GAIA Spontaneous Abortion and Ectopic Pregnancy Level of

Diagnostic Certainty Terminology. NCI Thesaurus. Code C128705.

A subset of terminology related to spontaneous abortion and ectopic pregnancy, developed by the Global Alignment of Immunization safety Assessment in pregnancy consortium to aid in monitoring and improving fetal and maternal outcomes. 